UNIVERSIDADE ESTADUAL DE FEIRA DE SANTANA

Autorizada pelo Decreto Federal no 77.496 de 27/04/76
Recredenciamento pelo Decreto n ${ }^{\circ} 17.228$ de 25/11/2016

PPPG

PRÓ-REITORIA DE PESOUISA E PÓS-GRADUAÇ̃̃o

COORDENAÇÃO DE INICIAÇÃO CIENTÍFICA

XXIII SEMINÁRIO DE INICIAÇÃO CIENTÍFICA DA UEFS

SEMANA NACIONAL DE CIENTÍFICA E TECNOLÓGICA - 2019

\title{
MANUTENÇÃO E VALIDAÇÃO DA COLEÇÃO DE CULTURA DE MICROFUNGOS DO PROGRAMA DE PESQUISA DE BIODIVERSIDADE DO SEMIARIDO BRASILEIRO- PPBIO
}

\author{
Maria das Graças de Santana Bispo ${ }^{1}$; Luís Fernando Pascholati Gusmão ${ }^{2}$ \\ 1. Bolsista PIBIC/CNPq, Graduanda em Licenciatura em Ciências Biológicas, Universidade Estadual de Feira de \\ Santana, e-mail: bio.maria.2014@gmail.com \\ 2. Orientador, Departamento de Ciências Biológicas, Universidade Estadual de Feira de Santana, e-mail: \\ lgusmao.uefs@gmail.com
}

PALAVRAS-CHAVE: Coleção de culturas; Microfungos; Semiárido.

\section{INTRODUÇÃO}

O Projeto de Pesquisa em Biodiversidade do semiárido (PPBio do SEMIÁRIDO) visa fomentar pesquisas que caracterizam a biodiversidade da região Semiárida Brasileira, das quais incluem ampliação de coleções biológicas de plantas, animais e também de fungos.

As coleções de cultura são importantes centro de armazenamento de espécies ex-situ, pois os organismos nelas mantidos servem como material de apoio a comunidade acadêmica, e também contribuem para a conservação de biodiversidade microbiológica (Canhos et al 1999). A manutenção dos isolados, tanto para fins, taxonômicos, sistemáticos e filogenéticos, bem como para interesses médico, industrial, farmacêutico, além do desenvolvimento de estratégias de preservação, entre outros, são importantes pois muitas espécies coletadas, podem não ser mais encontradas em seu ambiente natural, por conta dos impactos ambientais, que podem levar a perda de muitos microrganismos (Mello et al 2011).

De acordo com Figueiredo (2001), as coleções de culturas são importantes por depositarem amostras de microrganismos, mantendo-os vivos e viáveis por longos períodos sem variação de suas características genéticas. Essas coleções não são apenas depósitos, e sim centros que propiciam o acesso a diversos tipos de organismos por pesquisadores interessados a qualquer tempo (Abreu \& Tutunji, 2008). Desta forma, o este trabalho teve como objetivo de manutenção, validação e estudo morfológico 
(identificação/autenticação) dos espécimes depositados na Coleção de Cultura do Laboratório de Micologia (CCLAMIC).

\section{PROCEDIMENTOS METODOLÓGICOS}

Os procedimentos realizados para alcançar os objetivos foram realizados nas dependências do Laboratório de Micologia (LAMIC), localizado na Universidade Estadual de Feira de Santana. O LAMIC conta com microscópio, lupa, fluxo laminar, e autoclave, estufa de esterilização, vidrarias e outros reagentes necessários para o pleno desenvolvimento do trabalho proposto. Os métodos de reativação, preservação e validação utilizados estão descritos por Choi, et al. (1999), Figueiredo, (2001); Mello, (2011). Para o crescimento do fungo foi realizado a inoculação do esporo ou parte do micélio em placas de Petri, contendo meio de cultura nutritivo, que na sua maioria foi o CMA (Cenoura, amido de Milho, Agar). Após o crescimento e esporulação do espécime foi feito a identificação por meio de analises de lâminas em microscopia óptica, e comparação com a literatura pertinente do espécime. Após a autenticação e confirmação na literatura, o espécime foi novamente depositado na coleção.

De acordo com Cavalcante (2010), os métodos de preservação podem variar de acordo com as características do organismo de interesse. E são classificados em de curto, médio e longo prazo. Para a preservação foram utilizados os métodos de médio prazo: i) método de Castellani, que consiste na retirada de pedaços da cultura já validada, os quais são armazenados em frascos do tipo penicilina, contendo água destilada estéril e lacrados; ii) método de óleo mineral, que consiste em cobrir a cultura jovem com óleo mineral estéril em tubos de ensaio, não sendo recomendado camadas de óleo superior a $1 \mathrm{~cm}$ de altura, pois podem diminuir o tempo de viabilidade das culturas (Figueiredo, 2001). Os espécimes foram preservados em três frascos de Castellani e em cinco tubos com óleo mineral, e armazenados na CCLAMIC.

\section{RESULTADOS E DICUSSÃO}

A CCLAMIC conta atualmente com 2.224 acessos devidamente identificados. Semanalmente, são inseridos novos espécimes resultado dos trabalhos desenvolvidos no laboratório. No período de Agosto de 2018 à Julho de 2019, foram inseridos 94 novos acessos, que foram devidamente identificados e preservados na coleção. Paralelamente a inclusão, foram reativados, autenticados e reinseridos 31 espécimes, dos quais deu-se 
prioridade aos que estavam em menor número de réplicas e que estavam com mais de 3 anos de preservados (Sola, 2012). Na figura 1, estão representados os números de depósitos ao longo dos anos desde a fundação da coleção em 2006.

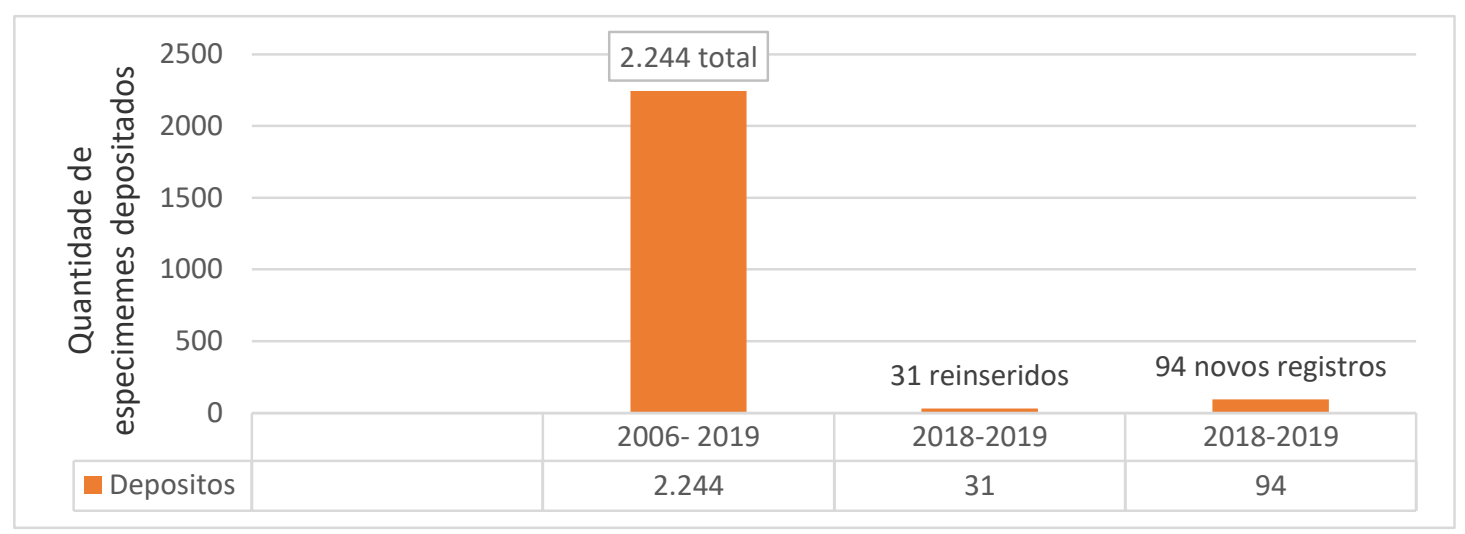

Figura 1. Período de depósitos ao longo dos anos. De 2006- 2019: 2.244; 2018-2019 reinseridos 31 espécimes; 2018 2019 deposito de 94 novos registros

Em uma coleção sugere-se a utilização de pelo menos dois métodos de preservação, pois nem sempre um método pode servir para todos os fungos (Mello, 2011), bem como os fungos possuem características intrínsecas e podem responder melhor a determinado método de preservação do que a outro, uma vez que o método de preservação deve proporcionar a viabilidade do fungo por um período mais longo possível (Figueiredo 2011). Os métodos de Castellani e óleo mineral, são de baixo custo, possibilitam maior viabilidade dos fungos cultivados por longos períodos, diminuindo o risco de contaminação por ácaros, e reduzem o número de repicagens constantes.

De acordo com Queiroz et al (2006) o número de fungos do semiárido já depositados em herbário e em coleções ainda e relativamente pouco quando comparado com o número de espécies conhecidas no mundo. Isso se deve, não pelo fato da inexistência dos fungos na região e sim pelo reduzido número de pesquisas sendo desenvolvidas nessas áreas. Desta forma, podemos afirmar que o número de registros está intimamente ligado a locais com pontos de coleta, ou seja, que tem pesquisas sendo desenvolvidas. $\mathrm{O}$ que ressalta a importância do investimento e fomento de projetos como o PPBio. No gráfico 2, a seguir, isso fica evidente, pois o número de espécimes depositados, é mais expressivo nas áreas com mais pontos de coletas. 


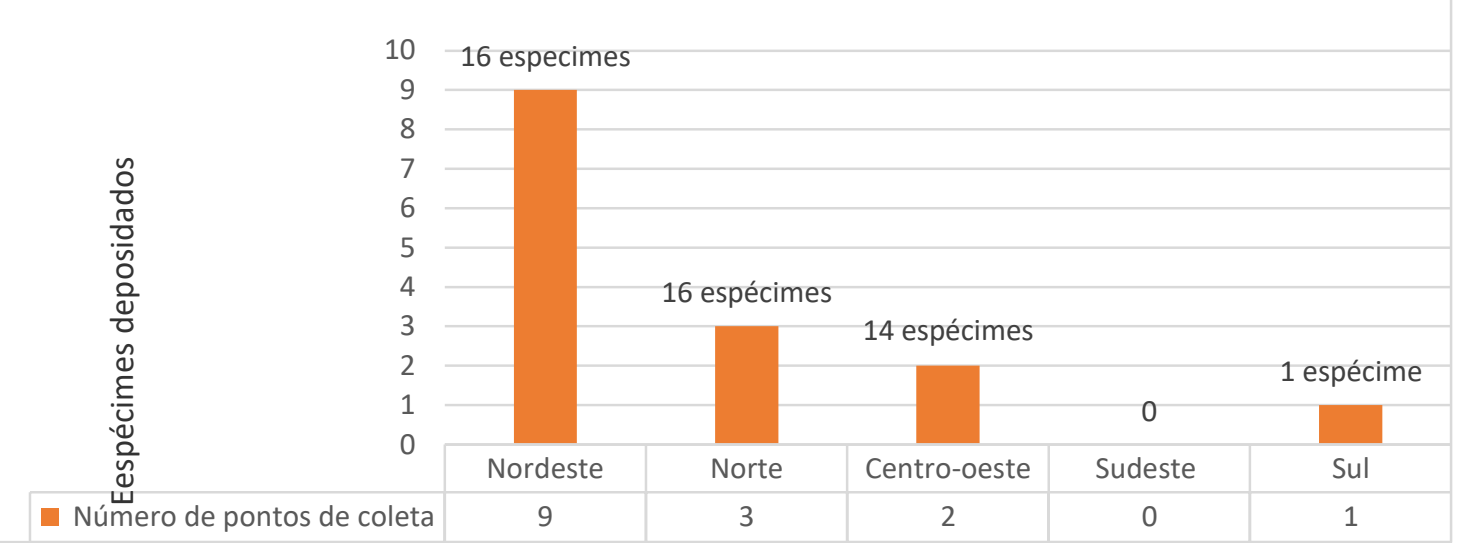

Figura 2. Relação das regiões do Brasil onde foram coletados os espécimens do CLAMIC.

\section{CONSIDERAÇÕES FINAIS}

Tendo em vista a importância da região semiárida, que apresenta poucos estudos desta natureza, e pela rápida perda de sua biodiversidade, resultado de ações antrópicas e do aquecimento global, a preservação de microfungos em coleções de cultura se torna uma estratégia para a conservação da micodiversidade presente nesta região.

\section{REFERÊNCIAS}

ABREU, M.M. V.; TUTUNJI, V.L. 2008. Implantação e manutenção da coleção de culturas de microorganismos do UniCEUB. Universitas: Ciências da Saúde, v. 2, n. 2 , p. 237-252.

CHOI, Y.W., HYDE, K.D. and HO, W.H. 1999. Single spore isolation of fungi. Fungal Diversity 3: 29-38.

CAVALCANTE, S.D.B. 2010. Aplicação de metodologias de preservação e caracterização de fungos na coleção de culturas do Instituto de Medicina Tropical de São Paulo. Faculdade de Medicina da Universidade de São Paulo, Dissertação.

CANHOS, V.P.; UMINO, C.; MANFIO, G.P.1999. Coleções de culturas de microrganismos. Biodiversidade do Estado de São Paulo, Brasil: síntese do conhecimento no final do século XX, v. 7, p. 81-101.

FIGUEIREDO, M.B. 2001. Métodos de preservação de fungos patogênicos. Instituto Biológico, São Paulo, v63,n 1⁄2, p. 73-82.

MELLO, S.C. M.; REIS, A.; SILVA, J.B.T. 2011. Manual de curadores de germoplasma-micro-organismos: fungos filamentosos. Embrapa Recursos Genéticos e Biotecnologia-Documentos (INFOTECA-E).

QUEIROZ, Luciano P., RAPINI, A., GIULIETTI, A. M. 2006. Rumo ao amplo Conhecimento da Biodiversidade so Semiárido Brasileiro. P. 1-144.

SOLA, M.C. et al. 2012. Manutenção de microrganismos: conservação e viabilidade. Enciclopédia biosfera, Centro Científico Conhecer - Goiânia, v.8, N.14; p. 1 398-1418. 
